\title{
Inactivation of Francisella tularensis Utah-112 on Food and Food Contact Surfaces by Ultraviolet Light
}

\author{
Christopher H. Sommers*, O. Joseph Scullen, George C. Paoli, and Saumya Bhaduri
}

U.S. Department of Agriculture, Agricultural Research Service, Eastern Regional Research Center, 600 East Mermaid Lane, Wyndmoor, Pennsylvania 19038, USA

\begin{abstract}
Francisella tularensis is the causative agent of tularemia, a plague-like illness that affects animals and humans, and has caused large illness pandemics in the last century. It has also been used as a biological warfare agent, and tularemia can be contracted through consumption of contaminated food and water. In this study the use of a U.S. Food and Drug Administration approved technology, $254 \mathrm{~nm}$ ultraviolet light (UV-C), to inactivate $F$. tularensis Utah112 (a rodent pathogen) on food and food contact surfaces was investigated. The $D_{10}$ value, the UV-C dose needed to inactivate one log of microorganism, was approximately $0.71 \mathrm{~mJ} / \mathrm{cm}^{2}$ on agar plates using a low UV-C intensity of $100 \mu \mathrm{W} / \mathrm{cm}^{2} / \mathrm{s}$. When a commercial UV-C conveyor was used $\left(5 \mathrm{~mW} / \mathrm{cm}^{2} / \mathrm{s}\right) 0.5 \mathrm{~J} / \mathrm{cm}^{2}$ inactivated $>7 \mathrm{log}$ CFU of $F$. tularensis Utah 121 on agar plates. At $0.5 \mathrm{~J} / \mathrm{cm}^{2} \mathrm{UV}-\mathrm{C}$ inactivated $>4 \log$ CFU of Utah-112 in beef, chicken, catfish, frankfurter, and bratwurst exudates inoculated onto stainless steel coupons, and $>7$ log CFU was eliminated at 1 $\mathrm{J} / \mathrm{cm}^{2}$ UV-C. Similar results were obtained when the exudates were inoculated onto high density polypropylene. Approximately 0.5 log CFU was inactivated on chicken breast, beef steak, and catfish fillets, and approximately 1.9 log CFU on frankfurters and bratwurst at a UV-C dose of $1 \mathrm{~J} / \mathrm{cm}^{2}$. These results indicate routine use of UV-C during food processing would provide workers and consumers some protection against $F$. tularensis.
\end{abstract}

Keywords: Francisella tularensis; Ultraviolet; Food; Food contact; Beef; Sausages; Chicken; Tilapia; Catfish

\section{Introduction}

Francisella tularensis is the bacterium responsible for a lethal plague-like illness in humans and animals known as tularemia. It has been responsible for large epidemics in Europe and Asia, and causes epizootic illnesses elsewhere in the world [1]. The utility of F. tularensis as a biological weapon was studied extensively by during World War II, and the microorganism may have used in that conflict for that purpose. During the Cold War F. tularensis was studied for its potential as a biological weapon [1,2]. Various scenarios have been developed by international health organizations to assess the health and economic impacts of a biological attack using F. tularensis [3,4]. F. tularensis is now considered a category A biological agent by the Centres for Disease Control and Prevention (CDC) [2].

Tulermia may be contracted through consumption of contaminated food and water and it is also possible to contract the disease by handling infected animals in a food processing environment [5]. Fortunately, the infectious dose required to cause Tulermia through consumption of contaminated foods may be higher than that required to cause infection by inhalation. The $\mathrm{LD}_{50}$ for mice given $F$. tularensis by gastric lavage was approximately $10^{6} \mathrm{CFU}$, much greater (>100,000 fold) than for intradermal injection or exposure by aerosol [6,7]. Human volunteers who consumed $10^{10} \mathrm{CFU}$ of $F$. tularensis in vaccine development studies required treatment with antibiotics to prevent imminent death [8]. Studies with primates indicated an oral dose of $10^{6}-10^{7} \mathrm{CFU}$ of $F$. tularensis required intervention with antibiotics to ensure survival [9]. Currently, there is very little information available regarding the utility of food safety intervention technologies to inactivate $F$. tularensis on food and food contact surfaces. The thermal $\mathrm{D}_{10}$ (the energy needed to inactivate one log of microorganism) of $F$. tularensis in infant formula and fruit juice has been determined [10]. High pressure (500 mPa, 2 min) inactivated $5 \log$ CFU of $F$. tularensis LVS in milk or orange juice [11]. Ultraviolet light inactivated $>7 \log \mathrm{CFU}$ of $F$. tularensis in distilled water at a UV-C dose of $4 \mathrm{~mJ} / \mathrm{cm}^{2}$ [12]. Ultraviolet light (UV-C, 254 $\mathrm{nm}$ ), a U.S. Food and Drug Administration (FDA) approved food safety intervention technology, can be used for decontamination of food and food contact surfaces [13]. Ultraviolet light exerts its bactericidal effect primarily through the formation of DNA adducts including cyclobutane pyrimidine dimers and 6-4 photoproducts, either killing the bacteria or rendering them unable to reproduce [14]. It has also been suggested as a possible intervention technology for mitigation of biological threats [15]. The technology is currently used by a number of industries, including the health care industry for the decontamination of hospital rooms, and is slowly beginning to see increased usage by the food industry [16-20]. Because the oral infectious dose, and $\mathrm{LD}_{50}$, for $F$. tularensis may be relatively high in comparison to exposure by aerosol, food safety intervention technologies which inactivate food borne pathogens may provide some protection against Tulermia for workers and consumers. The purpose of this study was to determine the effectiveness of UV-C for inactivating $F$. tularensis on the surface of solid agar growth medium, in food exudates (also known as drip or purge) placed on food contact surfaces, and on food.

\section{Materials and Methods}

\section{Francisella tularensis strains}

Francisella tularensis strains LVS, NIH B-38, and Utah-112 were obtained from BEI Resources (Manassas, VA). F. tularensis LVS and

\footnotetext{
${ }^{*}$ Corresponding author: Christopher H. Sommers, Research leader, Food Safety and Intervention Technologies Research Unit, Eastern Regional Research Center, USDA-ARS, 600 East Mermaid Lane, Wydnmoor, PA 19038, USA, E-mail: christopher.sommers@ars.usda.gov

Received September 13, 2012; Accepted October 27, 2012; Published November 05, 2012

Citation: Sommers CH, Scullen OJ, Paoli GC, Bhaduri S (2012) Inactivation of Francisella tularensis Utah-112 on Food and Food Contact Surfaces by Ultraviolet Light. J Food Process Technol S11-002. doi:10.4172/2157-7110.S11-002

Copyright: (c) 2012 Sommers $\mathrm{CH}$, et al. This is an open-access article distributed under the terms of the Creative Commons Attribution License, which permits unrestricted use, distribution, and reproduction in any medium, provided the original author and source are credited.
} 
NIH B-38 are attenuated strains which have been used for vaccine development, while Utah-112 is a rodent pathogen. F. tularensis was propagated on fluidthioglycolate medium containing $1 \%$ agar (FTG, BD Difco, Sparks, MD) at $37^{\circ} \mathrm{C}$ until ready for use. Liquid cultures for use in experiments were prepared by inoculating single colonies into 10 $\mathrm{ml}$ of freshly prepared FTG medium in sterile $50 \mathrm{ml}$ test tubes (Fisher Scientific, USA) and incubating $\left(37^{\circ} \mathrm{C}\right)$ in a shaking incubator $(150$ rpm) (New Brunswick, Inc., Model G24, Edison, NJ) for approximately $24 \mathrm{~h}$

\section{Food products}

Catfish fillets were obtained from the USDA-ARS Catfish Genetics Laboratory in Stoneville, Mississippi. Beef steaks, boneless skinless chicken breast fillets, frankfurters, and bratwurst were obtained from local retailers. The meat, poultry, and ready-to-eat meat products were gamma irradiated, to an absorbed dose of $10 \mathrm{kGy}$ to inactivate background microflora to less than $1 \mathrm{CFU} / \mathrm{g}$. Exudate (drip/purge) was obtained from the irradiated products by pouring excess liquid into sterile test tubes.

\section{Determination of $F$. tularensis $\mathrm{D}_{10}$ values on agar plates.}

Prior to determination of $\mathrm{D}_{10}$ values, media and growth conditions were evaluated for the recovery of $F$. tularensis from agar plate cultures and inoculated foods. Blood-Cystine-Dextrose agar, Mueller-Hinton agar and Fluid Thioglycollate (FTG) medium and incubation in either an aerobic or a $5 \% \mathrm{CO}_{2}$ environment were tested. Stationary phase cultures individually propagated and decimally diluted $(0.1 \mathrm{ml}$ culture in $0.9 \mathrm{ml}$ diluent) in Butterfield's Phosphate Buffer (BPB, Applied Research Institute, Newtown, CT, USA) using sterile borosilicate test dilutions were then plated on duplicate FTG agar plates which were then allowed to dry for approximately $30 \mathrm{~min}$. Exposures to UV-C were then carried using the source from a biological safety cabinet (NuAire, Inc., Model No. NU-425-800, Plymouth, MN)) at a UV-C intensity of approximately $100 \mu \mathrm{W} / \mathrm{cm} / \mathrm{s}$, for $10,20,30$, and $40 \mathrm{~s}$, to obtain UV-C doses of $1,2,3$, and $4 \mathrm{~mJ} / \mathrm{cm}^{2}$. The FTG plates were then incubated from $2-5$ days $\left(37^{\circ} \mathrm{C}\right)$ prior to enumeration of colony forming units (CFU). The agar plates for strain Utah-112 could be enumerated after 2-3 days, while strains LVS and NIH B-38 required 4-5 days incubation. The $\mathrm{D}_{10}$ values, the UV-C radiation doses needed to inactivate $90 \% \mathrm{~F}$. tularensis, were calculated from the enumeration data. The average bacterial population of the UV-C treated sample (N) was divided by the average population in the untreated control $\left(\mathrm{N}_{0}\right)$ to produce a survivor ratio $\left(\mathrm{N} / \mathrm{N}_{0}\right)$. Radiation $\mathrm{D}_{10}$-values were determined by calculating the reciprocal of the slope of the $\log \left(\mathrm{N} / \mathrm{N}_{0}\right)$ ratios versus the irradiation dose. Each experiment was conducted independently three times $(n=3)[21]$.

\section{Exposure inoculated foods and exudate to ultraviolet light (UV-C)}

A commercial food-grade UV-C conveyor (Reyco Systems, Inc. Meridan, Idaho) was used to treat exudates inoculated with F. tularensis on surfaces of stainless steel coupons, high density polypropylene (Oneida, Inc, Syracuse, NY), and the foods themselves [22,23]. The UV-C intensity was $5 \mathrm{~mW} / \mathrm{s} / \mathrm{cm}^{2}$, and 100 seconds of exposure provided a total UV-C dose of approximately $0.5 \mathrm{~J} / \mathrm{cm}^{2}$. The exudates were inoculated onto stainless steel or polypropylene coupon surfaces and were run through the conveyor either once or twice to obtain exposures of $0.5 \mathrm{~J} / \mathrm{cm}^{2}$ or $1.0 \mathrm{~J} / \mathrm{cm}^{2}$, respectively. Foods were passed through the conveyor up to 4 times to obtain the maximum UV-C dose of $2.0 \mathrm{~J} / \mathrm{cm}^{2}$. UV-C intensity was measured using a calibrated UVX
Radiometer (UVP, Inc, Upland, CA). The conveyor automatically rotated the frankfurters and bratwurst, which are cylindrical, during the exposure period. The temperature of the room was approximately $15^{\circ} \mathrm{C}$ during the exposure to UV-C, and food temperature did not increase to more than $20^{\circ} \mathrm{C}$ at the end of the process as measured using an infrared thermometer [24].

\section{UV-C inactivation of $F$. tularensis Utah-112 in exudate (drip)}

Exudates (drip/purge) were collected from the packages of irradiated food products. Overnight $F$. tularensis culture was then diluted $1 / 10$ in the exudates and mixed by vortexing for approximately 10 seconds. Following mixing, $0.1 \mathrm{ml}$ of exudate $\left(10^{7} \mathrm{CFU} / \mathrm{ml}\right)$ was pipeted onto a stainless steel coupon, which was then given either one or two passes through the UV-C conveyor $\left(0.5\right.$ and $\left.1.0 \mathrm{~J} / \mathrm{cm}^{2}\right)$. The stainless steel coupon was then placed in a sterile polynylon bag with $0.9 \mathrm{ml}$ of FTG medium, serially diluted in BPB, and $0.1 \mathrm{ml}$ spread into duplicate FTG agar plates. The plates were allowed to dry for approximately $30 \mathrm{~min}$ and then incubated for approximately 3 days at $37^{\circ} \mathrm{C}$ prior to enumeration of $\mathrm{CFU}$.

\section{UV-C inactivation of $F$. tularensis Utah-112 on foods}

Chicken, fish, and beef pieces (approximately $3 \mathrm{oz}$ ) were surface inoculated with $0.1 \mathrm{ml}(106 \mathrm{CFU} / \mathrm{ml})$ of a dilution of Utah-112 which was then spread on the food surface using a sterile inoculation loop, allowed to sit in a refrigerator $\left(4^{\circ} \mathrm{C}\right)$ of for $30 \mathrm{~min}$, and then passed through the conveyor to obtain the required UV-C doses $(0.25,0.5,1.0$ and $2.0 \mathrm{~J} / \mathrm{cm}^{2}$ ). Following exposure to UV-C food samples were placed in sterile polynylon bags (Uline, Inc. Philadelphia, PA) containing 100 $\mathrm{ml} \mathrm{BPB}$. The solution was then serially diluted in $9 \mathrm{ml} \mathrm{BPB}$ and two $0.1 \mathrm{~mL}$ aliquots per dilution were spread on FTG agar plates. The plates were then incubated in for $2-5$ days at $37^{\circ} \mathrm{C}$ prior to enumeration of bacterial colonies (Lab-Line Amb-Hi- Low Chamber, Melrose, IL, USA). Each experiment was conducted independently 3 times $(n=3)$.

\section{Results and Discussion}

To date there is a dearth of data regarding the ability of food safety intervention technologies, including UV-C, to inactivate the biothreat agent $F$. tularensis on food and food contact surfaces. Rose and O'Connell [12] found that a UV-C dose of $4 \mathrm{~mJ} / \mathrm{cm}^{2}$ inactivated F. tularensis LVS and NY98 by 6.6 and $8.7 \log$ CFU, respectively, when suspended in water, indicating a $\mathrm{D}_{10}$ of approximately $0.5-1 \mathrm{~mJ} / \mathrm{cm}^{2}$. In the current study, the ability of UV-C to inactivate $F$. tularensis on agar medium, food exudates on a food contact surfaces (stainless steel), and food was investigated. The study, in addition to determining inactivation kinetics in a typical laboratory setting, also investigated inactivation of $F$. tularensis using commercial ultraviolet light food decontamination equipment.

Initially the $\mathrm{D}_{10}$ value, the UV-C dose needed to inactivate one log of microorganism, of three F. tularensis strains Utah-112, LVS, and NIH B-38 were determined when inoculated onto thioglycollate agar plates. The $D_{10}$ values for those three strains are listed in table 1 , with Utah-112 being the most resistant to UV-C with a $\mathrm{D}_{10}$ of $0.71 \mathrm{~J} / \mathrm{cm}^{2}$. These results are at the low end of the range of $D_{10}$ reported for food borne pathogens and bacteria of $1.5-8 \mathrm{~J} / \mathrm{cm}^{2}$ as reported in other studies [25]. When the three $F$. tularensis strains were inoculated on agar plates and then run through the commercial UV-C conveyor $(0.5 \mathrm{~J} /$ $\mathrm{cm}^{2}, 5 \mathrm{~mW} / \mathrm{cm}^{2} / \mathrm{s}$ ) no colonies were recovered ( $>7 \log$ CFU reduction). We observed that $\mathrm{D}_{10}$ for Utah-112 could be determined in 2- 3 days while the $\mathrm{D}_{10}$ for LVS and NIH B-38 required incubation for 4-5 days 
for colony growth, enumeration, and $\mathrm{D}_{10}$ determination. In addition, Utah-112 gave more consistent results than LVS and NIB B 3-8 when recovering F. tularensis from actual food matrices. Therefore, Utah-112 was used for inactivation studies in food exudates.

Inactivation of pathogens on food and food contact surfaces is important as workers could come in contact with foodborne pathogens such as $F$. tularensis during the handling of foods or the cleaning and sanitation of equipment [5]. Inactivation of the bacteria prior to spraying and washing of equipment, as part of the equipment sanitation process, could mitigate the risk to plant workers from inhalation of $F$. tularensis aerosols. When F. tularensis Utah-112 was inoculated in beef, chicken, catfish, tilapia, frankfurter, or bratwurst exudates, which was then placed on the surface of stainless steel coupons, a UV-C dose of $0.5 \mathrm{~J} / \mathrm{cm}^{2}$ inactivated $4.56-5.10 \mathrm{log}$ CFU of F. tularensis Utah-112 (Figure 1A). Similar results were obtained when the microorganism was inoculated onto high density polypropylene (Figure 1B). The pathogen was reduced $>7$ log CFU (essentially eliminated) at a UV-C dose of $1.0 \mathrm{~J} / \mathrm{cm}^{2}$.

The inactivation of $F$. tularensis Utah-112 inoculated onto actual

\begin{tabular}{|c|c|c|}
\hline Strain & $\mathrm{D} 10(\mathrm{~mJ} / \mathrm{cm} 2)$ & $\mathrm{R} 2$ \\
\hline Utah-112 & $0.71 \mathrm{a}$ & 0.96 \\
\hline LVS & $0.62 \mathrm{~b}$ & 0.95 \\
\hline NIH B-38 & $0.64 \mathrm{~b}$ & 0.94 \\
\hline Mean (SEM) & $0.66(0.03)$ & N/A \\
\hline
\end{tabular}

Table 1: Ultraviolet light inactivation of Francisella tularensis on thioglycollate agar plates. Each experiment was conducted independently 3 times. $D_{10}$ values with the same superscript letters are statistically similar as determined by student's t-test $(p<0.05)$. Standard error of the mean (SEM) is shown in parenthesis.

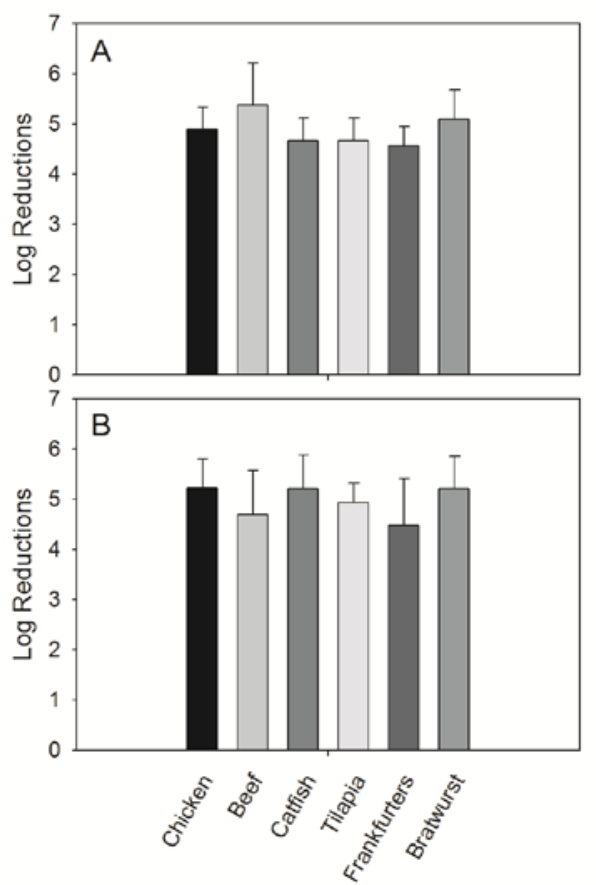

Figure 1: Inactivation of $F$. tularensis Utah-112 in food exudates inoculated onto: A) stainless steel; and B) polypropylene by ultraviolet light (UV-C). The UV-C dose was $0.5 \mathrm{~J} / \mathrm{cm}^{2}$. Each experiment was conducted independently 3 times. Standard error of the mean is shown as error bars. There was no difference in the mean log reductions as determined by ANOVA $(n=3, p>0.05)$.

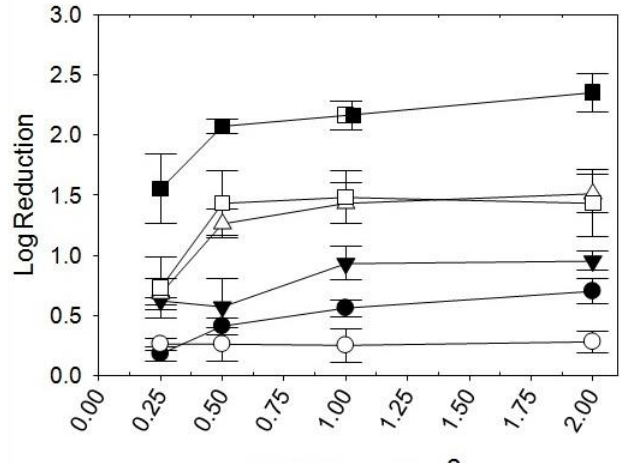

UV-C Dose $\left(\mathrm{J} / \mathrm{cm}^{2}\right)$

Figure 2: Inactivation of $F$. tularensis Utah-112 on foods by ultraviolet light (UV-C). UV-C doses for beef (o), chicken $(\bullet)$, catfish $(\boldsymbol{\nabla})$, tilapia $(\Delta)$, bratwurst $(\square)$, and frankfurters $(\square)$ were $0.25,0.5,1.0$ and $2.0 \mathrm{~J} / \mathrm{cm}^{2}$. Each experiment was conducted independently 3 times. Standard error of the mean is shown as error bars.

food products and exposed to UV-C $\left(0.25-2.0 \mathrm{~J} / \mathrm{cm}^{2}\right)$ (Figure 2). In each case the inactivation obtained was equal to or superior to those of other food borne pathogens including Escherichia coli O157:H7, Listeria monocytognes, Salmonella spp., Staphylococcus aureus, and Yersinia pestis using similar commercial grade equipment when inoculated products were treated with UV-C doses ranging 0.5 to 2.0 [23-25]. Similar results, although more inconsistent, were obtained with F. tularensis LVS and NIH B-38 (not reported). Although the dose exceeded that typically needed to inactivate $F$. tularensis on the surface of agar plates, higher intensities are typically required to inactivate microorganisms in the presence of particulate matter, UV absorbing organic materials, biofilms, or within tissue [26]. The tailing effect observed on actual meat and fish products has been described in previous studies, and can be attributed to shielding effects due to topological differences in food surface and food surface porosity $[26,27]$.

It has been suggested previously that UV-C light technology has potential for use to combat and mitigate acts of bioterrorism [15]. In this study it was determined that the sensitivity to UV-C was below 1 $\mathrm{J} / \mathrm{cm}^{2}$ for $F$. tularensis on agar surfaces, that $F$. tularensis was effectively eliminated from food contact surfaces when suspended on beef, poultry, and fish exudates and that inactivation of $F$. tularensis on actual beef, poultry and fish surfaces was consistent with inactivation for other food borne pathogens. Thus, UV-C light technology could mitigate the risk of $F$. tularensis contamination in a food processing environment.

\section{References}

1. Dennis DT, Inglesby TV, Henderson DA, Bartlett JG, Ascher MS, et al. (2001) Tularemia as a biological weapon: medical and public health management. JAMA 285: 2763-2773.

2. Khan AS, Morse S, Lillibridge S (2000) Public-health preparedness for biological terrorism in the USA. Lancet 356: 1179-1182.

3. WHO (1970) Tularemia. In: Health aspects of chemical and biological weapons World Health Organization, Geneva, SUI 105-107.

4. Kauffman AF, Meltzer MI, Schmid GP (1997) The economic impact of a bioterrorist attack:are prevention and post attack programs justifiable. Emerg Infect Dis 3: 83-94.

5. Pang ZC (1987) The Investigation of the first outbreak of tularemia in Shandong Peninsula. Zhonghua Liu Xing Bing Xue Za Zhi. 8: 261-263. 
Citation: Sommers CH, Scullen OJ, Paoli GC, Bhaduri S (2012) Inactivation of Francisella tularensis Utah-112 on Food and Food Contact Surfaces by Ultraviolet Light. J Food Process Technol S11-002. doi:10.4172/2157-7110.S11-002

Page 4 of 4

6. Jones R, Nicas M, Hubbard A, Sylvester M, Reingold A. (2005) The infectious dose of Francisella tularensis (tularemia). Appl Biosafety 10: 227-239.

7. KuoLee R, Zhao X, Austin J, Harris G, Conlan JW, et al. (2007) Mouse model of oral infection with virulent type A Francisella tularensis. Infect Immun 75: 1651-1660.

8. Hornick RB, Dawkins AT, Eigelsbach HT, Tulis JJ (1966) Oral tularemia vaccine in man. Antimicrob Agents Chemother (Bethesda) 6: 11-14.

9. Tulis JJ, Eigelsbach HT, Hornick RB (1969) Oral vaccination against tularemia in the monkeys. Proc Soc Exp Biol Med 132: 893-897.

10. Day JB, Trujillo S, Hao YY, Whiting RC (2008) Thermal resistance of Francisella tularensis in infant formula and fruit juices. J Food Prot 71: 2208-2212.

11. Schlesser JE, Parisi B (2009) Inactivation of Yersinia pseudotuberculosis 197 and Francisella tularensis LVS in beverages by high pressure processing. $J$ Food Prot 72: 165-168.

12. Rose LJ, O'Connell H (2009) UV light inactivation of bacterial biothreat agents. Appl Environ Microbiol 75: 2987-2990.

13. Code of Federal Regulations (CFR). 2005. Irradiation in the production, processing and handling of food. Code of Federal Regulations. 21 (179): 450456 .

14. Reardon JT, Sancar A (2005) Nucleotide excision repair. Prog Nucleic Acid Res Mol Biol 79: 183-235.

15. Brickner PW, Vincent RL, First M, Nardell E, Murray M, et al. (2003) The application of ultraviolet germicidal irradiation to control transmission of airborne disease: bioterrorism countermeasure. Public Health Rep 118: 99-114.

16. Anonymous (2009) Preventing infection with UV light. Western Mass. hospital sets new industry standard for patient care and cleanliness Available at: http:// www.cooley- dickinson.org/node/624. Accessed March 1, 2012.

17. Yoosook W, Kongtip P, Chantanakul S (2009) Design and development of ultraviolet germicidal irradiation device for controlling airborne bacteria in the workplace. J Med Assoc Thai 7: S142-S147.

18. Nerandzic MM, Cadnum JL, Pultz MJ, Donskey CJ (2010) Evaluation of an automated ultraviolet radiation device for decontamination of Clostridium difficile and other healthcare-associated pathogens in hospital rooms. BMC Infect Dis 10:197.

19. Rutala WA, Gergen MF, Weber DJ (2010) Room decontamination with UV radiation. Infect Control Hosp Epidemiol 31: 1025-1029.

20. Koutchma T. (2008) UV light for processing foods. IUVA News 10: 24-29.

21. Diehl J (1995) Safety of irradiated food. (2nd edn). Marcel Dekker, New York New York. p. 93-98.

22. Sommers $\mathrm{CH}$, Sites JE, Musgrove M (2010) Ultraviolet light (254 nm) inactivation of pathogens on foods and stainless steel surfaces. J Food Safety 30: $470-479$.

23. Koutchma T, Forney L, Moraru C (2009) Microbial inactivation by UV light. In: Ultraviolet light in food technology. Taylor \& Francis CRC Press, New York USA.

24. Sumner SS, Wallner-Pendleton EA, Froning GW, Stetson LV (1996) Inhibition of Salmonella typhimurium on agar medium and poultry skin by ultraviolet energy. J Food Prot 59: 319-321.

25. Stermer RA, Lasater-Smith M, Brasington CF (1987) Ultraviolet Radiation-An effective bactericide for fresh meat. J Food Prot 50: 108-111.

26. Kim T, Silva JL, Chen TC (2002) Effects of UV irradiation on selected pathogens in peptone water and on stainless steel and chicken meat. J Food Prot 65 $1142-1145$.

27. Gardner DW, Shama G (2000) Modeling UV-induced inactivation of microorganisms on surfaces. J Food Prot 63: 63-70. 